\title{
Design and Implementation of Potentiostat with Standalone Signal Generator for Vanillylmandelic Acid Biosensors
}

\author{
Chun-Yueh Huang* \\ Department of Electrical Engineering, National University of Tainan, \\ No. 33, Sec. 2, Shu-Lin Street, Tainan 70005, Taiwan
}

(Received September 7, 2016; accepted November 21, 2016)

Keywords: potentiostat, electrochemical biosensors, signal processing circuit

In this paper, a portable potentiostat with a standalone signal generator is proposed for the signal processing of vanillylmandelic acid (VMA) biosensors. The proposed potentiostat primarily consists of two microprocessors: one is used to design the programmable waveform generator, and the other is used to measure the current of the biosensors. It can perform general electrochemical analysis functions, such as cyclic voltammetry (CV), linear sweep voltammetry (LSV), differential pulse voltammetry (DPV), amperometry (IT), and potentiometry (E). In the experiment, we adopt a VMA biosensor to verify the performance of the proposed potentiostat. The experimental results show that the proposed potentiostat has the merits of good accuracy, larger range of detected sensor current, low cost, and high portability.

\section{Introduction}

Vanillylmandelic acid (VMA), which is an end-stage metabolite of the catecholamines epinephrine and norepinephrine, is found in urine and can be used to diagnose an adrenal gland tumor called pheochromocytoma, namely, a tumor of catecholamine-secreting chromaffin cells. ${ }^{(1)}$ The aim of this study is to design a potentiostat for VMA biosensors to enable the detection of renal diseases.

In this work, we adopt an electrochemical approach to design VMA biosensors that yield an output change in the form of potential or current in accordance with the VMA concentration. ${ }^{(2)}$ In every electrochemical sensor, a potentiostat ${ }^{(3)}$ is needed to maintain electrochemical stability in the sensor. In other words, the potentiostat is an indispensable device for electrochemical sensors. To date, two types of potentiostat have been frequently used: one is a commercial potentiostat designed for research in the laboratory, and the other is a specific potentiostat developed for a particular sensor. The former is sophisticated, expensive, and nonportable, and the latter is simple, inexpensive, and portable. In light of the low cost and portability, the latter seems to be more popular for researchers to study.

Previously, many researchers devoted themselves to developing single-chip potentiostats to reduce the chip's size. Turner et al. first presented a basic complementary metal-oxidesemiconductor (CMOS) integrated potentiostat, ${ }^{(4)}$ Liao et al. presented a CMOS potentiostat with glucose sensor for wireless contact-lens tear glucose monitoring, ${ }^{(5)}$ Nazari et al. proposed a 96-channel potentiostat for on-die microsensors, ${ }^{(6)}$ Zuo et al proposed a low-power $1 \mathrm{~V}$ ${ }^{*}$ Corresponding author: e-mail: cyhun@gm2.nutn.edu.tw http://dx.doi.org/10.18494/SAM.2017.1461 
potentiostat for glucose sensors, ${ }^{(7)} \mathrm{Kim}$ and Ko proposed a 1.2 V analog front-end integrated circuit (IC) for glucose monitoring, ${ }^{(8)}$ and Huang et al. proposed ICs for the monolithic implementation of a voltammeter potentiostat with a large range of dynamic current (5 nA to $1.2 \mathrm{~mA}$ ) and short conversion time $(10 \mathrm{~ms}){ }^{(9)}$ However, the above potentiostats cannot be directly used because they need to be integrated with other devices as a complete system for actual applications.

For the sake of direct applications, in this paper, we adopt off-the-shelf components to design the potentiostat by means of a system approach. In the past, we proposed two portable potentiostats to mimic the commercial potentiostat's function to reduce the cost, using SoCbased microprocessors and off-the-shelf circuit components to implement potentiostats on printed-circuit boards (PCBs). ${ }^{(10,11)}$ However, the two portable potentiostats had the small problem of the scan rate mismatch in cyclic voltammetry, because they used a microprocessor to deal with signal generation and current measurement at the same time. In order to solve such a problem, in this study, we adopt two microprocessors to design a portable potentiostat with a standalone signal generator for the signal processing of VMA biosensors: one is used to construct the programmable waveform generator, and the other is used to measure the current of the biosensors. The design specifications of the proposed potentiostat are described as follows: the range of the programmable scan potentials $\left(V_{W R}\right)$ is between -1.6 and $1.6 \mathrm{~V}$ under $1 \mathrm{mV}$ resolution, the minimum programmable scan rate is $5 \mathrm{mV} / \mathrm{s}$, and the range of the measured current $\left(I_{W C}\right)$ is from $16 \mathrm{~mA}$ under $78 \mu \mathrm{A}$ resolution to $160 \mathrm{nA}$ under $78 \mathrm{pA}$ resolution. With the proposed potentiostat, the electrochemical measurements of biosensors can be performed outside the laboratory.

\section{The Design of Potentiostat}

\subsection{Principle of potentiostat operation}

A potentiostat is an electronic device used to maintain electrochemical stability in a biosensor and to convert the biosensor's output into an analog signal. ${ }^{(12)}$ A biosensor is composed of a working electrode (W), a reference electrode (R), and a counter electrode (C). The working electrode attached with the biomolecular probes can generate the reaction of the target. Figure 1(a) is a diagram of the potentiostat operation, which can be implemented using the electronic components shown in Fig. 1(b).

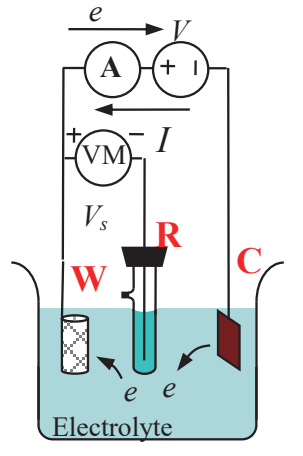

(a)

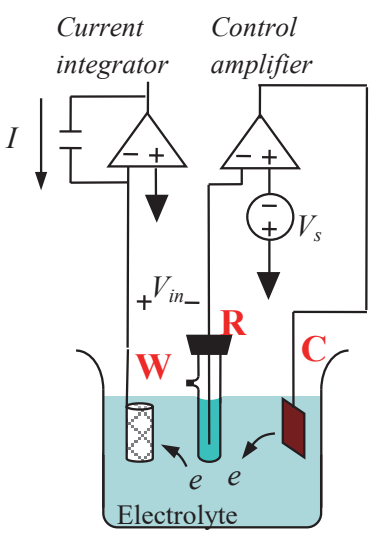

(b)

Fig. 1. (Color online) Potentiostat operation: (a) concept diagram and (b) implementation using electronic components. 


\subsection{Design of a portable potentiostat}

Figure 2 shows the circuit diagram of a portable potentiostat. The proposed potentiostat is mainly constructed using two mixed-signal microprocessors C8051F005 (MCU1and MCU2), a voltage amplifier, an autorange current-to-voltage converter, a voltage follower, an operational amplifier, a DC-DC power converter, and an secure digital (SD) memory card. The cost of the proposed potentiostat is $\$ 25$, where the C8051F005 microprocessor is $\$ 7.5$. In comparison with the cost of a commercial potentiostat, which is more than $\$ 3000$, the proposed potentiostat is much cheaper. On the other hand, in comparison with the cost stated in Refs. 10 and 11, the proposed potentiostat is merely $\$ 7.5$ higher, but it has more electrochemical analysis functions than those described in Refs. 10 and 11, and a better accuracy of the scan rate.

In Fig. 2, MCU2 is used to communicate with a PC via a USB interface. MCU1 is used to generate the required signal waveform via a 12-bit digital-to-analog converter (DAC) after the experimental parameters of the potentiostat are stored in the SD memory card. The signal range of the DAC, $0-2.4 \mathrm{~V}$, will be amplified to $0-3.2 \mathrm{~V}$ by the voltage amplifier to extend the scan range of the potentiostat. During the experiment, the potentiostat is controlled by the microcontroller. The power supply can be provided by a USB charger without using an AC power supply. This design approach can increase the portability and ability of standalone operation.

In the potentiostat, the autorange current-to-voltage converter, which contains a transimpedance amplifier and a set of resistors $(10 \mathrm{M} \Omega, 1 \mathrm{M} \Omega, 100 \mathrm{k} \Omega, 10 \mathrm{k} \Omega, 1 \mathrm{k} \Omega$, and $100 \Omega$ ), is used to measure the current flowing through the solution between the working and the counter electrodes. It can be used to automatically convert the sensor's current range from $\pm 160 \mathrm{nA}$ to $\pm 16 \mathrm{~mA}$ by the microprocessor by adjusting the resistance value. For considering the mismatch of resistors values, a calibration table is programmed in LabVIEW to correct the measured currents converted from different resistors. This calibration table is established using different values of precision resistors acting as biosensor electrodes to perform cyclic voltammetry analysis.

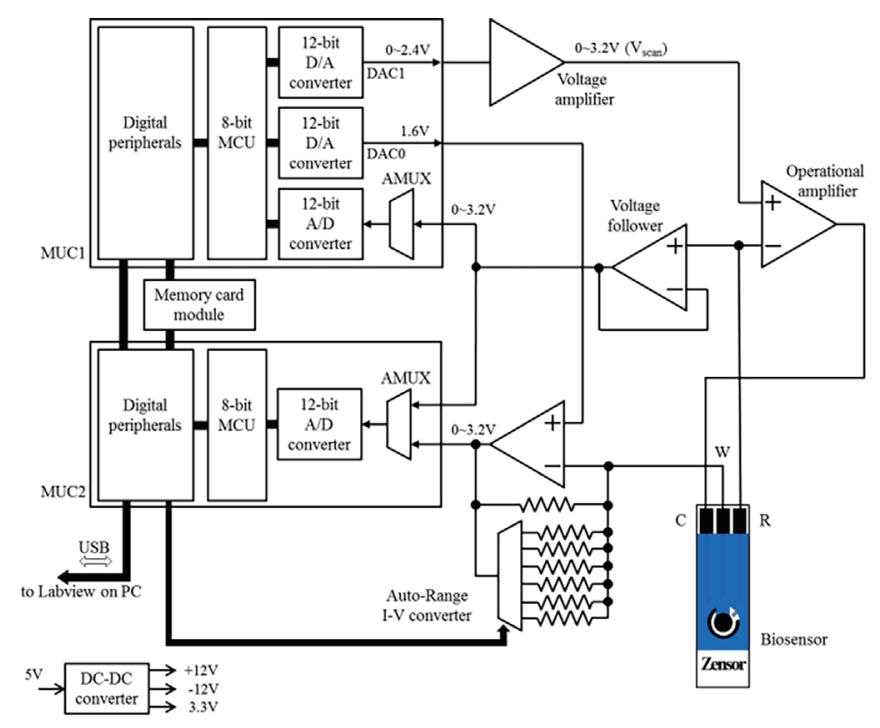

Fig. 2. (Color online) Circuit diagram of a portable potentiostat with standalone signal generator. 
The C8051F005 chip is an 8051-based CPU with 12-bit DACs, 12-bit analog-to-digital converters (ADCs), and digital peripherals. According to the specifications of the C8051F005 chip, the output range of DAC is between 0 and $2.4 \mathrm{~V}$, and thus DAC can generate only positive potential. In order to realize the function of cyclic voltammetry, the potentiostat must generate a sweep potential in both forward and reverse directions. Thus, two 12-bit DACs, DAC1 and DAC0, are used to generate a scan potential between 0 and $3.2 \mathrm{~V}$, and a $1.6 \mathrm{~V}$ offset potential, respectively. The potential of the working electrode $\left(V_{W}\right)$ is set by the voltage on the operational amplifier's virtual "ground" node of $1.6 \mathrm{~V}$. On the basis of the preset scan potential, the microprocessor generates a triangular signal, and then DAC1 transforms it into a triangular analog voltage between 0 and $2.4 \mathrm{~V}$. Furthermore, this voltage will be amplified to 0 and $3.2 \mathrm{~V}$, which acts as the setting voltage $\left(V_{\text {set }}\right)$. In the operational amplifier, a negative feedback loop controls the potential of the reference electrode $\left(V_{R}\right)$ to be equal to the setting voltage $\left(V_{\text {set }}\right)$. By this approach, the scan voltage $V_{W R}$ will be obtained in the range of -1.6 to $1.6 \mathrm{~V}$.

The design specifications of the proposed potentiostat are described as follows: the range of the programmable scan potentials $\left(V_{W R}\right)$ is between -1.6 and $1.6 \mathrm{~V}$ under $1 \mathrm{mV}$ resolution, the minimum programmable scan rate is $5 \mathrm{mV} / \mathrm{s}$, and the autorange of the measured current $\left(I_{W C}\right)$ is from $\pm 16 \mathrm{~mA}$ in the resolution of $8 \mu \mathrm{A}$ to $\pm 160 \mathrm{nA}$ in the resolution of $80 \mathrm{pA}$.

The scan rate is defined as

$$
S R=\frac{\Delta V}{\Delta T}=\frac{V_{2}-V_{1}}{T_{2}-T_{1}}
$$

where $\Delta V$ is the step of scan potentials and $\Delta T$ is the time step between two neighbour potentials. In Eq. (1), the minimum of the step of scan potentials is $1 \mathrm{mV}$, and the minimum $\Delta T$ will be limited by the operation speed of DAC and ADC in MCU, which is about $2 \mathrm{~ms}$. Hence, the minimum programmable scan rate in the proposed potentiostat is set as $5 \mathrm{mV} / \mathrm{s}$.

\subsection{Autorange current-to-voltage converter}

In the potentiostat, the autorange current-to-voltage converter is used to measure the biosensor's current. To choose a suitable resistance value in the set of resistors, a sequential search algorithm is used to find the suitable resistor for current-to-voltage conversion. Figure 3 shows the flow chart of the resistor selection process. On the basis of the sequential search algorithm, at first, the resistance value of $100 \Omega$ is selected, and then the converted voltage is measured using the ch-4 ADC in MCU2. The measured voltage is checked to determine whether it is in the range of

$$
1.44<V_{c h 4}<1.76 \text {. }
$$

If the converted voltage is in the range of Eq. (2) (it means that the presented resistor value is too small to be used), then the resistance value will be enlarged tenfold. The foregoing procedure will be continued until the range of the converted voltage satisfies the condition of Eq. (3) or until the resistor values reaches $10 \mathrm{M} \Omega$.

$$
V_{c h 4}<1.44 \text { or } V_{c h 4}>1.76
$$




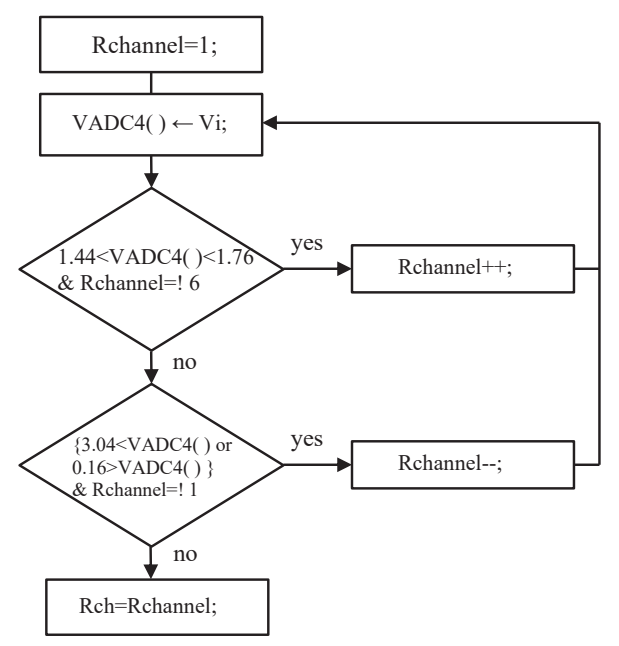

Fig. 3. Flow chart of the resistor selection process in the autorange current-to-voltage conversion.

When a suitable resistance value is found, the resistance value will be recorded and sent to the next cycle of current-to-voltage conversion. In the new conversion cycle, according to the variation of the biosensor current, the previous conversion resistor may not be suitable for the present current conversion. Hence, the conversion resistor will be changed to have either a larger resistance value if the condition of Eq. (2) is satisfied or a smaller resistance value if the condition of Eq. (4) is satisfied.

$$
V_{c h 4}>3.04 \text { or } V_{c h 4}<0.16
$$

After the resistor selection process is completed, as shown in Fig. 3, Rch will represent the selected suitable resistor to perform the current-to-voltage conversion.

\subsection{Personal user interface}

The experiment is controlled by a PC running the LabVIEW software, via a USB interface. The personal user interface is shown in Fig. 4. In Fig. 4, the electrochemical analysis functions include cyclic voltammetry, linear sweep voltammetry, differential pulse voltammetry, amperometry, and potentiometry. By choosing the cyclic voltammetry mode, the experimental parameter setting table, real-time cell potential, real-time sensor current, and voltammogram diagram will be shown. Before the experiment, the user must set some experimental parameters, such as the range of scan (set the "initial" and "final" settings), the scan rate, and the scan cycles. The scan rate is determined by the number of increments in the output voltage (called "steps") and the number of seconds fixed in each step.

During the experiment, the LabVIEW program controls the experimental procedure and collects the measured data from the potentiostat. Before the LabVIEW program plots the cyclic voltammogram, a digital 'weighted averaging filter' ${ }^{\text {(13) }}$ is used to smooth the measured current signal. The algorithm of the weighted averaging filter is expressed as

$$
Y(t)=\frac{[(L-1) \times Y(t-1)] \times X(t)}{L} .
$$




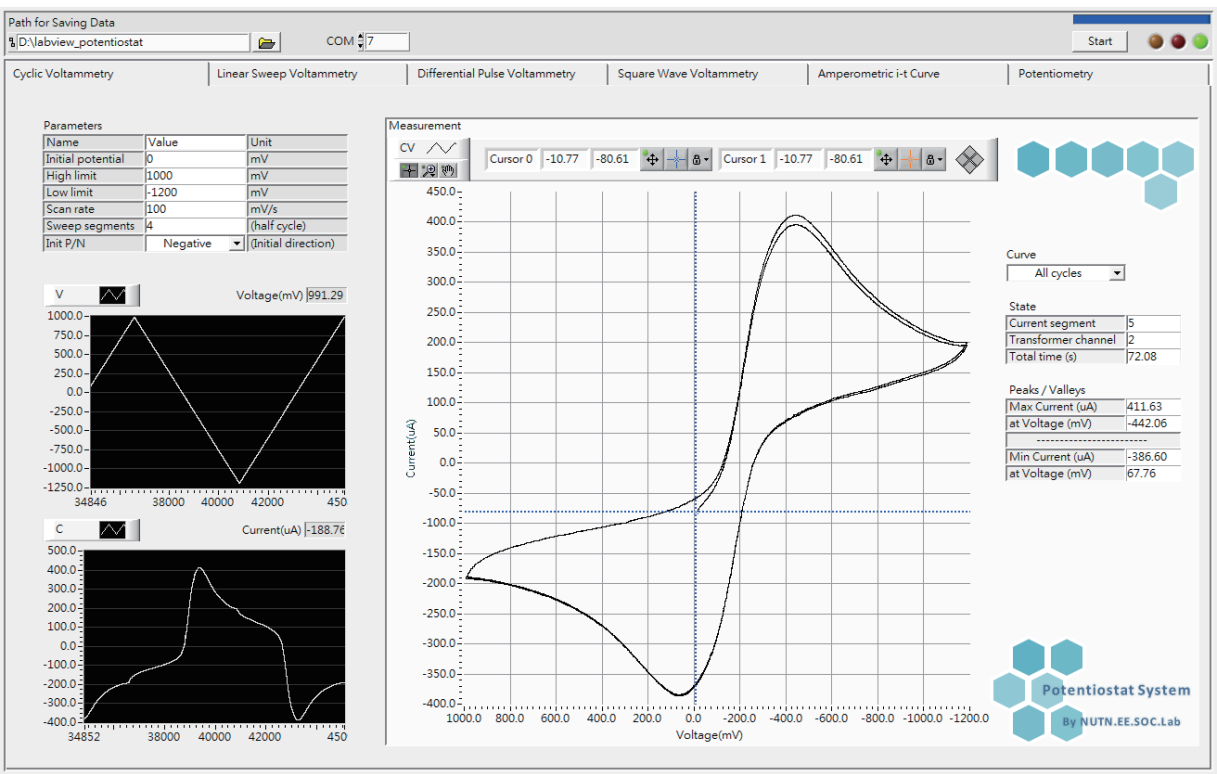

Fig. 4. (Color online) Labview operation interface of PC.

In Eq. (5), $X(t)$ is the value of the new input sample, $Y(t-1)$ is the value of the previous output, $Y(t)$ is the value of the present output, and $L$ (set as 15 in our system) is the weighted factor of the filter setting.

\subsection{SD memory card}

The SD memory card is used to store the experimental parameters of the potentiostat. In the SD memory card, there are two alternative communication protocols: SD and serial peripheral interface (SPI). For the memory card to communicate with the microcontroller, the SPI communication protocol, which is one of the built-in communication protocols of C8051F005, is used to reduce the design-in effort to minimum. The SPI interface communication can be completed by connecting the signals of CS, CLK, DataIn, and DataOut in the SD memory card with the SPI interface of C8051F005. The communication between the microcontroller and the memory card is controlled by the microcontroller (MCU2). The FAT16 format is used to store data in the SD card. The measured data of each experiment is stored in one text file and can be retrieved and analyzed using a PC to plot the experimental diagram.

\subsection{Fabrication of electrochemical VMA biosensor}

In this work, we utilized a three-electrode configuration with a working electrode, a $\mathrm{Ag} / \mathrm{AgCl}$ reference electrode, and a platinum counter electrode (CE) to construct the VMA biosensor. The VMA-imprinted polymer, which acted as the sensing element on the working electrode, with a corresponding shape to the target molecules, is recognized by selective binding, whereas interferences may not fit the binding site for the recognition. The concentration of VMA will be determined in accordance with the variation of the biosensor current. 
VMA and poly (ethylene-co-vinyl alcohol) (EVAL) with 27, 32, 38, and $44 \mathrm{~mol} \%$ ethylene (product Nos. 414077, 414093, 414085, and 414107) were purchased from Sigma-Aldrich (St. Louis, MO). The preparation of VMA-imprinted and nonimprinted (NIP) EVAL-coated working electrodes is briefly described as the following three steps: (1) $5 \mu \mathrm{L}$ of EVAL solution [EVAL/dimethyl sulfoxide $(\mathrm{DMSO})=0.1 \mathrm{wt} \%]$ mixed with and without $1 \mathrm{mg} / \mathrm{mL}$ of target molecules on the working electrode of a screen-printed electrode (Dropsens, Spain), (2) evaporate solvent in an oven at 60 ${ }^{\circ} \mathrm{C}$ for $9 \mathrm{~h}$ to completely remove DMSO, and then (3) remove the template molecules by rinsing in $20 \mathrm{~mL}$ of $1 \mathrm{wt} \%$ sodium dodecylsulfate (SDS) for $15 \mathrm{~min}$ and then deionized water for $15 \mathrm{~min}$, repeated three times. All electrodes are immersed and equilibrated with phosphate buffered saline (PBS) overnight before being used. In this work, VMA-imprinted poly (ethylene-co-vinyl alcohol) is coated on a commercial three-electrode screen-printing chip, as illustrated in Fig. 5.

\section{Experimental Results}

Differential pulse voltammetry (DPV), a highly sensitive electroanalytical approach with the characteristic of determining the redox properties of extremely small amounts of compounds in electrolyte solutions, is used to measure the signals of a VMA biosensor. A series of regular voltage pulses are superimposed on the stair step potential to a working electrode, and the current is immediately measured before each potential changes. The plot of the function of current difference corresponding to the potential of the pulse is a differential pulse voltammogram.

When we use the EVALs containing different mole percents of ethylene for the molecular imprinting of VMA, we find that the EVAL containing $32 \mathrm{~mol} \%$ of ethylene has a higher electrochemical response. The differential pulse voltammetry measurements for the VMA biosensor are performed in $10,25,50$, and $100 \mathrm{ng} / \mathrm{ml} \mathrm{VMA}$ solutions, and the scanned voltage is between -0.4 and $+0.4 \mathrm{~V}$. The differential pulse voltammetry profile of the molecularly imprinted polymer (MIP) electrode obtained from the VMA solution using the proposed potentiostat is shown in Fig. 6(a), in which we can see that VMA is reduced when $-40 \mathrm{mV}$ is applied. The maximum current difference is

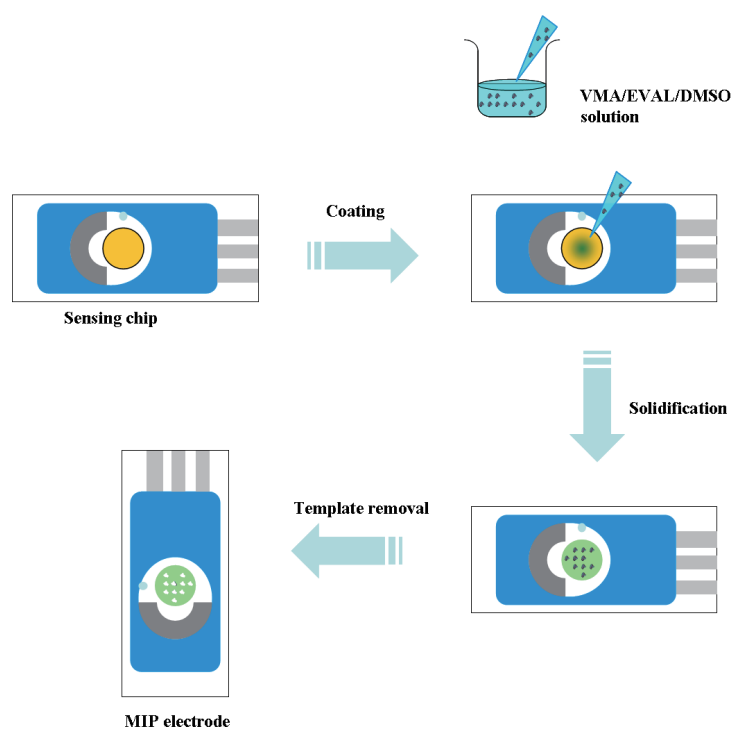

Fig. 5. (Color online) Preparation of VMA-imprinted EVAL-coated working electrode. 


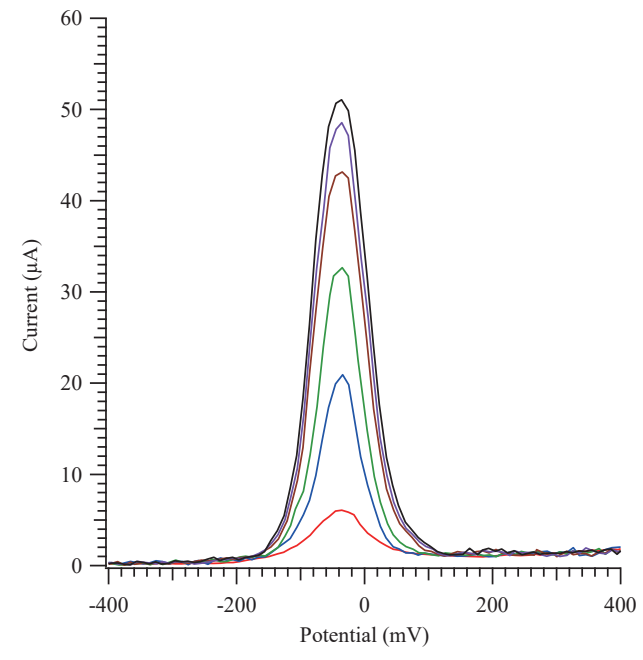

(a)

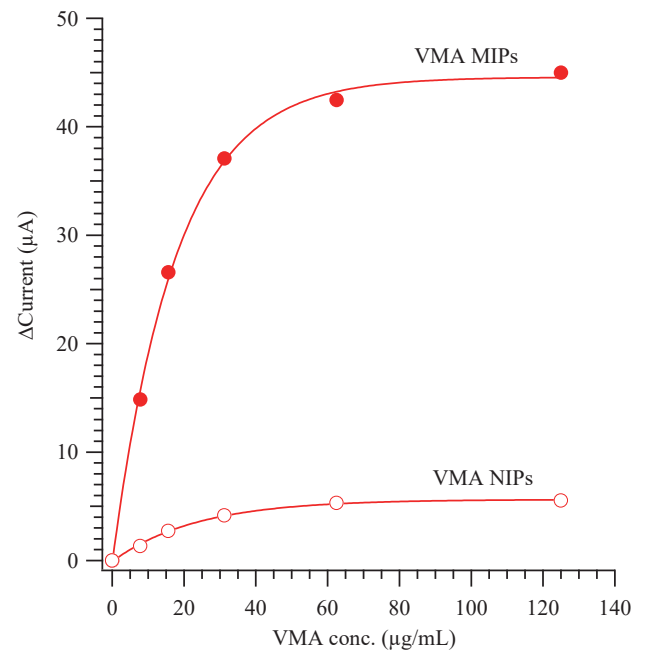

(b)

Fig. 6. (Color online) (a) Differential pulse voltammogram of the VMA-imprinted biosensor measured using the proposed potentiostat and (b) current difference for the VMA-imprinted EVAL-coated electrode plotted against different concentrations of VMA solution.

Table 1

Performance of different potentiostats.

\begin{tabular}{|c|c|c|c|c|c|}
\hline & $\begin{array}{l}\text { Detected sensor } \\
\text { current range }\end{array}$ & $\begin{array}{c}\text { Current } \\
\text { sensitivity }\end{array}$ & $\begin{array}{l}\text { Supply voltage } \\
\text { (V) }\end{array}$ & $\begin{array}{l}\text { Implement } \\
\text { approach }\end{array}$ & $\begin{array}{l}\text { Functions of } \\
\text { electrochemical analysis }\end{array}$ \\
\hline Turner et al. ${ }^{(4)}$ & $100 \mathrm{nA} \rightarrow 3.5 \mu \mathrm{A}$ & $100 \mathrm{nA}$ & \pm 5 & Chip & IT \\
\hline Liao et al. ${ }^{(5)}$ & $1 \mathrm{nA} \rightarrow 1 \mu \mathrm{A}$ & $1 \mathrm{nA}$ & 1.8 & Chip & IT \\
\hline Nazari et al. ${ }^{(6)}$ & $24 \mathrm{pA} \rightarrow 350 \mathrm{nA}$ & $24 \mathrm{pA}$ & 3.3 & Chip & IT \\
\hline Zuo et al..$^{(7)}$ & $70 \mathrm{nA} \rightarrow 2.6 \mu \mathrm{A}$ & $70 \mathrm{nA}$ & 1 & Chip & IT \\
\hline $\mathrm{Kim}$ and $\mathrm{Ko}^{(8)}$ & $260 \mathrm{pA} \rightarrow 7.5 \mu \mathrm{A}$ & $260 \mathrm{pA}$ & 1.2 & Chip & IT \\
\hline Huang et al. ${ }^{(9)}$ & $5 \mathrm{nA} \rightarrow 1.2 \mathrm{~mA}$ & $5 \mathrm{nA}$ & 3.3 & Chip & IT \\
\hline Huang et al..$^{(10)}$ & $100 \mathrm{nA} \rightarrow 100 \mu \mathrm{A}$ & $100 \mathrm{nA}$ & \pm 5 & System & $\mathrm{CV}, \mathrm{IT}$ \\
\hline Huang et al..$^{(1)}$ & $5 \mathrm{nA} \rightarrow 12 \mathrm{~mA}$ & $5 \mathrm{nA}$ & \pm 12 & System & $\mathrm{CV}, \mathrm{IT}$ \\
\hline This work & $78 \mathrm{pA} \rightarrow 16 \mathrm{~mA}$ & $78 \mathrm{pA}$ & \pm 12 & System & CV, LSV, DPV, IT, E \\
\hline
\end{tabular}

around $50 \mu \mathrm{A}$ when the VMA concentration is higher than $60 \mu \mathrm{g} / \mathrm{mL}$. Figure $6(\mathrm{~b})$ shows the current difference for the VMA-imprinted EVAL-coated electrode in accordance with different concentrations of the VMA solution. The saturated response for MIPs is about 8-fold higher than that of NIP electrodes. Finally, the high electrochemical response of approvimately $2 \mu \mathrm{A} /(\mu \mathrm{g} / \mathrm{mL}$ VMA) for the VMA-imprinted EVAL is obtained. From the experimental results, we can observe that the accuracy of the proposed potentiostat is as good as that of a commercial potentiostat.

Table 1 shows the proposed design and the potentiostats in the literature and their detected sensor current range, current sensitivity, supply voltage, implement approach, and functions of electrochemical analysis. In Table 1, we can observe that the proposed potentiostat has the maximum number of electrochemical analysis functions, and the maximum detected sensor current processing ability owing to the use of autorange current-to-voltage conversion. Moreover, 
the proposed potentiostat is highly suitable for the signal processing of different electrochemical biosensors and it can imitate the functions of commercial potentiostats.

\section{Conclusions}

In this paper, we proposed a portable potentiostat designed with a standalone signal generator for a VMA biosensor. It is also suitable for the signal processing of other types of electrochemical biosensors. Moreover, we successfully verified its performance by making it the major part of the VMA biosensor. The experimental results showed that the performance of the proposed potentiostat is as good as those of commercial ones. In addition, the proposed potentiostat has the merits of a larger range of detected sensor current, low cost, and high portability. In the future, the proposed potentiostat can be further used in conjunction with other biosensors for home-care system application.

\section{Acknowledgments}

This work was supported in part by the Ministry of Science and Technology, Republic of China, under Grants No. MOE 104-2221-E-024-017 and 105-2221-E-024-021. The author would also like to thank Mr. Ming-Feng Sun for the system implementation, and his co-worker, Professor HungYin Lin, from the Department of Chemical and Materials Engineering, National University of Kaohsiung, for providing a VMA biosensor for system verification.

\section{References}

1 M. J. Magera, A. L. Thompson, D. Matern, and P. Rinaldo: Clin. Chem. 49 (2003) 825.

2 S. Alegret and A. Merkoci: Electrochemical Sensor Analysis (Elsevier Science, B. V., 2007) p. 50.

3 F. Shahrokhi, K. Abdelhalim, D. Serletis, P. L. Carlen, and R. Genov: IEEE Trans. Biomed. Circuits Syst. 4 (2010) 149.

4 R. B. F. Turner, D. J. Harrison, and H. P. Baltes: IEEE J. Solid-State Circuits 22 (1987) 473.

5 Y. T. Liao, H. Yao, A. Lingley, B. Parviz, and B. P. Otis: IEEE J. Solid-State Circuits 47 (2012) 335.

6 M. H. Nazari, M. J. Hamed, L. Leng, A. Guenther, and R. Genov: IEEE Trans. Biomed. Circuits Syst. 7 (2013) 338.

7 L. Zuo, S. K. Islam, I. Mahbub, and F. Quaiyum: IEEE Trans. Circuits Syst. II 62 (2015) 204.

8 J. Kim and H. Ko: IEEE Sens. J. 16 (2016) 6517.

9 C. Y. Huang, I. J. Chao, J. L. Thomas, H. W. Wei, Y. F. Liang, B. D. Liu, M. H. Lee, and H. Y. Lin: Biosens. Bioelectron. 67 (2015) 208.

10 C. Y. Huang, M. J. Syu, C. H. Chang, Y. S. Chang, B. D. Liu, and T. C. Chou: Biosens. Bioelectron. 22 (2007) 1694.

11 C. Y. Huang, T. C. Tsai, J. L. Thomas, M. H. Lee, B. D. Liu, and H. Y. Lin: Biosens. Bioelectron. 24 (2009) 2611.

12 P. M. Levine, P. Gong, R. Levicky, and K. L. Shepard: IEEE J. Solid-State Circuits 43 (2008) 1859.

13 M. K. Ozkan, M. I. Sezan, and A. M. Tekalp: IEEE Trans. Circuits Syst. Video Technol. 3 (1993) 277. 


\section{About the Author}

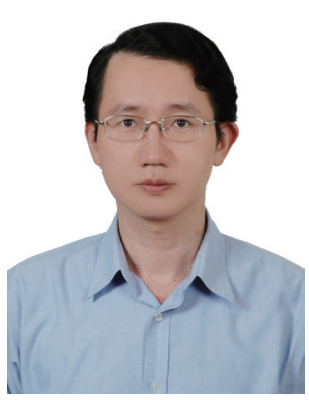

Chun-Yueh Huang was born in Taichung, Taiwan, Republic of China, on March 24, 1967. He received a B.S. degree in industrial education from the National Chang Hwa Normal University, Chang Hwa, Taiwan in 1991, and M.S. and Ph.D. degrees, both in Electrical Engineering, from the National Cheng Kung University, Tainan, Taiwan, in 1993 and 1997, respectively. Since 2005, he has been on the faculty of the National University of Tainan, where he is currently a Professor in the Department of Electrical Engineering. During 1999-2005, he was an Associate Professor in the Department of Electronic Engineering of the Kan Shan University. His current research includes signal processing of biosensors, VLSI design, and analog IC design. 